\title{
The association between platelet transfusion and mortality in patients with sepsis-induced thrombocytopenia: a propensity score-matching analysis
}

\section{Aiming Zhou ( $\nabla$ zhouaiming@wzhospital.cn )}

The First Affiliated Hospital of Wenzhou Medical University https://orcid.org/0000-0002-7308-1951

\section{Shanshan Wu}

The First Affiliated Hospital of Wenzhou Medical University

\section{Qin Chen}

The First Affiliated Hospital of Wenzhou Medical University

\section{Lili Chen}

The First Affiliated Hospital of Wenzhou Medical University Jingye Pan

The First Affiliated Hospital of Wenzhou Medical University

\section{Research}

Keywords: Platelet transfusion, sepsis-induced thrombocytopenia, propensity score matching, mortality.

Posted Date: May 5th, 2021

DOI: https://doi.org/10.21203/rs.3.rs-313903/v2

License: (c) (1) This work is licensed under a Creative Commons Attribution 4.0 International License.

Read Full License 


\section{Abstract}

Background: Thrombocytopenia is common among sepsis patients. Platelet transfusion is frequently administered to increase platelet counts but its clinical impacts remains unclear in sepsis-induced thrombocytopenia. Therefore, we intended to explore the association between platelet transfusion and mortality in patients with sepsis-induced thrombocytopenia.

Methods: The study was based on the Medical Information Mart for Intensive Care (MIMIC) III database. Sepsis patients were divided into two groups: platelet transfusion group (PT group) and no platelet transfusion group (NPT group). The primary outcome was 28-day mortality, and secondary outcomes were the length of ICU stay (LOS-ICU) and survival days at 28 days. Propensity-score matching was used to reduce the imbalance.

Results: 1733 patients were included: 296 patients were in the PT group and 1437 in the NPT group. Overall, 655 patients died by day 28 . After propensity score matching, 294 paired patients constituted each group. 28-day mortality did not decrease in PT patients comparing with NPT patients $(120(40.54 \%)$ vs. $535(37.23 \%)$ deaths; $p=0.29)$. LOS-ICU was similar between the PT group and the NPT group (5.84(2.68-11.78) vs. 4.94(2.18-12.72); $p=0.44)$. After confounders were adjusted, it showed no difference between the PT group and the NPT group in 28-day mortality (hazard ratio [HR], 1.28;95\% confidence interval [CI], $0.96-1.17 ; \mathrm{p}=0.09$ ) or LOS-ICU (odds ratio [OR], 0.09; 95\% Cl, $-1.45-1.62 ; \mathrm{p}=0.91$ ). Survival days at 28 days showed no difference between the groups according to Kaplan-Meier survival estimates. After propensity score matching, platelet transfusion was also not associated with 28-mortality or LOSICU.

Conclusions: In the propensity score-matched analysis, it showed that platelet transfusion maybe not associated with 28-day mortality and the length of ICU stay in patients with sepsis-induced thrombocytopenia.

\section{Background}

Sepsis is the most common disease in the intensive care unit (ICU), defined as a life-threatening syndrome of organ dysfunction which was caused by the dysregulated host response to severe infection ${ }^{1}$, and it has been considered a major cause of health loss worldwide. According to a recent scientific publication, there were about 48.9 million cases and 11 million sepsis-related deaths worldwide in 2017 , which accounted for almost $20 \%$ of all global deaths ${ }^{2}$.

Clinically, platelet count decrease is common among patients with sepsis who are admitted to the ICU. The incidence of sepsis-induced thrombocytopenia is around $25 \%$ on ICU admission ${ }^{3}$ and approximately $55 \%$ during the hospital stay ${ }^{4}$. Studies have confirmed that platelets play a crucial role in inflammatory balance, immune responses, tissue repair, and regeneration, beyond their important role in hemostasis and thrombosis ${ }^{5-7}$. Based on recent studies, thrombocytopenia is closely associated with multiple organ 
dysfunction syndromes, prolonged ICU stay, and high mortality in sepsis patients ${ }^{8}$. Besides, thrombocytopenia is an early prognostic marker for ICU patients in the first $24 \mathrm{~h}$ of septic shock onset ${ }^{9}$. And non-resolution of thrombocytopenia is associated with increased 28-day mortality in this population 3 .

In general, after controlling the infection and improving the patient's conditions, sepsis-induced thrombocytopenia is gradually returning to be normal. Recently, several studies have showed that recombinant human thrombopoietin (rhTPO) can lead to the quick recovery of the platelet count and improve the prognosis of patients with sepsis-induced severe thrombocytopenia ${ }^{10-12}$. And platelet transfusion is the most common clinical therapy to increase platelet counts. In theory, one standard unit dose of platelet transfusion can elevate platelet counts by $2010^{9} / \mathrm{L}$. However, platelet transfusion is limited in clinical practice and has a rigorous indication in sepsis patients ${ }^{13,14}$ because of resource scarcity, transfusion-related immune, and infectious complications. Thus, large prospective clinical trials are restricted in the exploration of the platelet transfusion impact on sepsis-induced thrombocytopenia.

According to a recent large registry study, it showed that platelet transfusion was not associated with the increased risk of death in critically ill patients ${ }^{15}$. Nonetheless, there is no large study based on platelet transfusion in severe sepsis-induced thrombocytopenia investigating whether platelet administration could improve the prognosis of sepsis patients. In this study, we aimed to determine the potential association between platelet transfusion and clinical outcomes including 28-day mortality and the length of ICU stay.

\section{Methods}

\section{Database}

This was a retrospective study based on an online international database, Medical Information Mart for Intensive Care III (MIMIC III), compromising the information of 46,520 critically ill patients who have been admitted to the Beth Israel Deaconess Medical Center from 2001 to $2012^{16}$. All the patients in the database were de-identified, and the need for informed consent was waived. One author (AZ) obtained access to this database (certification number 35752875 ) and was responsible for data extraction.

\section{Study population and definitions}

Septic patients with a platelet count level $\leq 5010^{9} / \mathrm{L}$ were eligible for inclusion in our study. And sepsis was defined according to the third sepsis definition ${ }^{1}$, which was extracted as suspected infection and an acute change in cases that total Sequential Organ Failure Assessment (SOFA) score $\geq 2$ points. For patients who were readmitted to the ICU, only the first ICU admissions were included. For patients younger than 18 years or older than 89 years were excluded. The primary outcome was 28 -day mortality. The secondary outcome was the length of ICU stay (LOS-ICU). 


\section{Propensity score matching}

PSM was used to minimize the imbalance of the confounding factor between the PT and the NPT groups. A one-to-one nearest neighbor matching algorithm was applied with a caliper width of 0.02 in our study. The following variables were selected to generate the propensity score: age, gender, SOFA, Simplified Acute Physiology score II (SAPS II), platelet count, platelet transfusion, diabetes mellitus, hypertension, chronic pulmonary, congestive heart failure, cancer.

\section{The management of missing data}

Variables with missing data are common in the MIMIC III database. For C-reactive protein, serum lactate, albumin, and procalcitonin values, more than $20 \%$ were missing and were removed from this analysis. For other continuous variables with missing values less than $5 \%$, the missing values were replaced by the mean or median values.

\section{Statistical analysis}

Continuous variables were depicted as means with standard deviations or medians with interquartile ranges (IQRs), as appropriate. Student's t test, analysis of variance, Wilcoxon rank-sum test, or KruskalWallis test were used, as appropriate. Categorical data were shown as frequencies and proportions, and they were compared using the X2 test. The association between platelet transfusion and 28-day mortality was determined by logistic regression including the baseline as the covariate and the group as a fixed factor. The survival outcomes comparisons between the groups were analyzed by the log-rank test. PSM was used to minimize the imbalance between groups. A two-tailed test was performed, and $p<0.05$ was considered to indicate statistical significance. All statistical analyses were performed using the $\mathrm{R}$ package (version 3.6.3).

\section{Results}

\section{Baseline characteristics}

The data of 1733 patients were included. The flow chart of patient selection is presented in Fig. 1. The overall 28 -day mortality rate was $38.9 \%$. The comparisons of the baseline characteristics are listed in Table 1. Patients in the PT group were younger than those in the NPT group ( $58.17 \pm 14.56$ vs. $61.00 \pm 15.14, p=0.001)$. The platelet count was significantly lower in patients with platelet transfusion $(22.37 \pm 12.84$ vs. $29.60 \pm 13.35, p<0.001)$. The SOFA score on admission was similar in the PT group and the NPT group $(8.81 \pm 3.84$ vs. $8.79 \pm 3.98, p=0.81)$. Patients in the PT group were more likely to combine with congestive heart failure ( $87(29.39 \%)$ vs. $311(21.64 \%), p=0.004)$, while more patients in the NPT group were complicating with chronic pulmonary $(29(9.80 \%)$ vs. $239(16.63 \%), p=0.003)$. The mortality of 28 days had no significant difference between the PT group and the NPT group (120(40.54\%) vs. 535(37.23\%), $p=0.29)$. 
On univariable analysis of risk of 28-day mortality was similar between the PT group and the NPT group $(\mathrm{HR}, 1.15 ; 95 \% \mathrm{Cl}, 0.89-1.48 ; \mathrm{p}=0.29)$. After confounders(platelet count and SOFA score) were adjusted, it revealed that platelet transfusion was also not associated with the overall 28-day mortality (HR, 1.28; $95 \% \mathrm{Cl}, 0.96-1.71 ; \mathrm{p}=0.09$ ) or LOS-ICU(OR, 0.08; $95 \% \mathrm{Cl},-1.45-1.61 ; \mathrm{p}=0.92)$ (Table 2). Also, there was no significant difference on the survival days at 28 days between the groups, according to Kaplan-Meier survival estimates (Figure 2)

\section{Outcomes after propensity score matching}

After PSM, 294 cases from each group were matched by a 1:1 matching algorithm (Table 3). To assess the overall quality of the matched sample, the standardized difference of the means and the ratio of the variances between the propensity scores of both groups were compared, and the propensity scores between the groups was also inspected. There was no significant difference between the two matched groups with regards to all eleven covariates(age, gender, SOFA, SAPSII, platelet count, diabetes mellitus, hypertension, chronic pulmonary, congestive heart failure, cancer) (Table 3). Among the 294 propensitymatched pairs, we found that the 28-day mortality had no evidently difference between the groups $(119(40.48 \%)$ vs. $107(36.40 \%), p=0.309)$, and LOS-ICU showed the similar results $(5.73(2.68-11.76)$ vs. 5.90(2.33-14.26), $p=0.606$ ) (Table 3).

\section{Discussion}

The present study demonstrates that platelet transfusion is not associated with 28-day mortality or LOSICU in sepsis patients with severe thrombocytopenia. This result was robust in the PSM analysis after adjustment for covariates including platelet counts and SOFA scores. According to our findings, it seems that platelet transfusion is not a reasonable choice to rescue sepsis-induced thrombocytopenia for improving the prognosis of sepsis patients with severe thrombocytopenia.

A low platelet count, thrombocytopenia, commonly occurs in sepsis patients. According to previous research, nearly $35 \%$ to $59 \%$ of patients with sepsis develop thrombocytopenia ${ }^{17,18}$, which has been recognized as an independent risk factor for mortality and a marker for disease severity ${ }^{19}$. Moreover, it is also an important index to evaluate the prognosis of patients ${ }^{20}$. Sepsis patients with a low platelet count or dynamic thrombocytopenia show a poor prognosis and increased mortality ${ }^{21}$. The mechanisms of sepsis-induced thrombocytopenia are complex and probably involve various factors. For instance, endothelial dysfunction is a major consequence of sepsis and plays a crucial role in platelet activation and consumption ${ }^{22}$. This activation which results in aggregation is increased locally by cytokine production ${ }^{23}$. Besides, altered thrombopoiesis and/or hemophagocytosis is the major causes of thrombocytopenia, which would be potentiated by sepsis mediators ${ }^{24}$. In addition, fluid resuscitation and surgical operation may have an influence on platelet count.

Sepsis patients with platelet counts less than $5010^{9} / \mathrm{L}$ are considered to have sepsis-induced thrombocytopenia ${ }^{25}$, which has a high mortality and poor prognosis. It has been reported that non- 
resolution of thrombocytopenia was associated with increased 28-day mortality, instead of thrombocytopenia itself ${ }^{3}$. Currently, there is no effective treatment for this condition. Infection control, organ support therapy and immune response regulation remain the mainstream treatments. In recent years, recombinant human thrombopoietin (rhTPO) was reported improving platelet count and reducing platelet transfusion possibility among patients with severe sepsis and thrombocytopenia in a prospective study ${ }^{10}$. In another research, it suggested that the rescue therapy with rhTPO could rapidly lead to a recovery of the platelet count, increase survival days and reduce the 28-day mortality in sepsis patients with severe thrombocytopenia ${ }^{12}$. Nevertheless, Yu Liu et al. found that rhTPO is efficacious in increasing the patients' platelet counts, resulting in a shorter ICU stay time $(9.20 \pm 5.38$ vs $10.88 \pm 6.82, p=0.047)$ for patients with severe thrombocytopenia or patients with severe sepsis, while there was no significant difference in 28 -days mortality ( rhTPO group: $25.0 \%$ vs. control group: $34.1 \%, p=0.158$ ) between the two groups $^{26}$. Therefore, whether patients with sepsis-induced thrombocytopenia can benefit from rhTPO therapy still remains a question according to the controversial results.

Platelet transfusion is a regular clinical practice in thrombocytopenic patients for preventing or treating hemorrhages. Approximately 1,937,000 platelet component transfusions are given in the United States in $2017^{27}$. There are some evidences suggesting that platelet transfusion is associated with adverse effects including infection ${ }^{28}$. Some experts believe that conventional platelet transfusion therapy may worsens patient's procoagulant and anticoagulant disorders. However, the other study noted that platelet transfusion was not associated with increased mortality or infective complications after first cardiac surgery ${ }^{29}$. A recent publication by Ning $S$ and colleagues reported that platelet transfusions were not associated with increased risk of death in critically ill patients neither in ICU (HR, 0.78; 95\% $\mathrm{Cl}, 0.60-1.02$; $\mathrm{p}=0.41)$ nor in hospital $(\mathrm{HR}, 0.89 ; 95 \% \mathrm{Cl}, 0.68-1.09 ; \mathrm{p}=0.41)^{14}$. In our study, we found nearly the same result among sepsis patients with thrombocytopenia, that platelet transfusion was not associated with increased risk of 28-day mortality or LOS-ICU. Regarding to our negative finding, patients with sepsisinduced thrombocytopenia may not benefit from platelet transfusion, which may also put patients into a potential risk of infection ${ }^{28}$. Therefore, it may be reasonable to avoid unnecessary transfusions.

In present study, there are still several limitations. Firstly, as a retrospective design, the adjustment of relevant but missing data was not allowed. Although we did perform propensity score matching to reduce the imbalance, estimation of the propensity score could only be based on the acquirable data. Secondly, bacteria species and sources were not recorded in our data and the purpose of platelet transfusion was also unknown. Thus, these two aspects could not be included in the analysis. Thirdly, patients who have been administrated with $\beta$-lactam or sulfa antibiotics, which may result in antibiotic-induced thrombocytopenia, did not excluded in this study. Lastly, well-organized prospective randomized clinical trials are required to analyze the role of platelet transfusion in sepsis-induced thrombocytopenia and identify patients most likely to benefit from platelet transfusion.

\section{Conclusions}


In our study, we found that platelet transfusion maybe not associated with 28-day mortality and the length of ICU stay in patients with sepsis-induced thrombocytopenia. And further prospective studies will be needed in the future to address the benefits and harms of platelet transfusion in sepsis patients with thrombocytopenia, to distinguish different subtypes of sepsis for which platelet transfusions would be of benefit, and to develop optimal platelet transfusion thresholds to better guide clinical treatment.

\section{Abbreviations}

MIMIC III: Medical Information Mart for Intensive Care III; PT group: Platelet Transfusion group; NPT group: No Platelet Transfusion group; ICU: Intensive Care Unit; LOS-ICU: length of ICU stay; SOFA: Sequential Organ Failure Assessment; SAPS II: Simplified Acute Physiology Score II; Cl: Confidence interval; HR: hazard ratio; OR: odd ratio; rhTPO: recombinant human thrombopoietin; PSM: propensity score matching; IQRs: interquartile ranges.

\section{Declarations}

\section{Acknowledgements}

We thank Dr. Xianwei Zhang (The First Affiliated Hospital of Wenzhou Medical University, Wenzhou, Zhejiang, China) and Dr. Jiejie Cai (The First Affiliated Hospital of Wenzhou Medical University, Wenzhou, Zhejiang) for their help in this revision.

\section{Funding}

Not applicable

\section{Availability of data and materials}

The datasets are available in the MIMIC III database

(https://physionet.org/works/MIMICIIIClinicalDatabase/files/).

\section{Authors' contributions}

AZ designed the study, extracted the data and performed all statistical analyses. SW wrote the draft of the manuscript. JP, QC, LC review the data analysis and interpretation, and revised the manuscript for the final version. All authors read and approved the final manuscript.

\section{Ethics approval and consent to participate}

The MIMIC III database was approved by the Massachusetts Institute of Technology (Cambrige, MA) and Beth Israel Deaconess Medical Center (Boston, MA) and consent was obtained for the original data collection. Therefore, the ethical approval statement and informed consent were waived for this manuscript. 


\section{Consent for publication}

Not applicable

\section{Competing interests}

The authors declare that they have no competing interests.

\section{Author details}

${ }^{1}$ Department of Intensive Care Unit, The First Affiliated Hospital of Wenzhou Medical University, Wenzhou, Zhejiang 325000, China.

${ }^{2}$ Medical Research Center, The First Affiliated Hospital of Wenzhou Medical University, Wenzhou, Zhejiang 325000 , China.

\section{References}

1. Singer M, Deutschman CS, Seymour CW, Shankar-Hari M, Annane D, Bauer M, et al. The Third International Consensus Definitions for Sepsis and Septic Shock (Sepsis-3). JAMA. 2016;315(8):801-10.

2. Rudd KE, Johnson SC, Agesa KM, Shackelford KA, Tsoi D, Kievlan DR, et al. Global, regional, and national sepsis incidence and mortality, 1990-2017: analysis for the Global Burden of Disease Study. Lancet. 2020;395(10219):200-211.

3. Venkata C, Kashyap R, Farmer JC, Afessa B. Thrombocytopenia in adult patients with sepsis: incidence, risk factors, and its association with clinical outcome. J Intensive Care. 2013;1(1):9.

4. Sharma B, Sharma M, Majumder M, Steier W, Sangal A, Kalawar M. Thrombocytopenia in septic shock patients-a prospective observational study of incidence, risk factors and correlation with clinical outcome. Anaesth Intensive Care. 2007;35(6):874-80.

5. Cedervall J, Hamidi A, Olsson A-K. Platelets, NETs and cancer. Thromb Res. 2018;164:S148-52.

6. Sreeramkumar V, Adrover JM, Ballesteros I, Cuartero MI, Rossaint J, Bilbao I, et al. Neutrophils scan for activated platelets to initiate inflammation. Science. 2014;346(6214):1234-8.

7. Jenne CN, Kubes P. Platelets in inflammation and infection. Platelets. 2015;26(4):286-92.

8. Greco E, Lupia E, Bosco O, Vizio B, Montrucchio G. Platelets and Multi-Organ Failure in Sepsis. Int J Mol Sci. 2017;18(10):2200.

9. Thiery-Antier N, Binquet C, Vinault S, Meziani F, Boisramé-Helms J, Quenot J-P. Is Thrombocytopenia an Early Prognostic Marker in Septic Shock?. Crit Care Med. 2016;44(4):764-72.

10. Wu Q, Ren J, Wu X, Wang G, Gu G, Liu S, et al. Recombinant human thrombopoietin improves platelet counts and reduces platelet transfusion possibility among patients with severe sepsis and thrombocytopenia: A prospective study. J Crit Care. 2014;29(3):362-6. 
11. Zhou Z, Feng T, Xie Y, Huang P, Xie H, Tian R, et al. The effect of recombinant human thrombopoietin (rhTPO) on sepsis patients with acute severe thrombocytopenia: a study protocol for a multicentre randomised controlled trial (RESCUE trial). BMC Infect Dis. 2019;19(1):780.

12. Zhou Z, Feng T, Xie Y, Zhang X, Du J, Tian R, et al. Prognosis and rescue therapy for sepsis-related severe thrombocytopenia in critically ill patients. Cytokine. 2020;136:155227.

13. Rhodes A, Evans LE, Alhazzani W, Levy MM, Antonelli M, Ferrer R, et al. Surviving Sepsis Campaign: International Guidelines for Management of Sepsis and Septic Shock: 2016. Crit Care Med. 2017;45(3):486-552.

14. Slichter SJ. Evidence-based platelet transfusion guidelines. Hematology Am Soc Hematol Educ Program. 2007;172-8.

15. Ning S, Liu Y, Barty R, Cook R, Rochwerg B, lorio A, et al. The association between platelet transfusions and mortality in patients with critical illness. Transfusion. 2019;59(6):1962-1970.

16. Johnson AEW, Pollard TJ, Shen L, Lehman L-WH, Feng M, Ghassemi M, et al. MIMIC-III, a freely accessible critical care database. Sci Data. 2016;3:160035.

17. Levi M, Löwenberg EC. Thrombocytopenia in critically ill patients. Semin Thromb Hemost. 2008;34(5):417-24.

18. Sakr Y, Vincent J-L, Ruokonen E, Pizzamiglio M, Installe E, Reinhart K, et al. Sepsis and organ system failure are major determinants of post-intensive care unit mortality. J Crit Care. 2008;23(4):475-83.

19. Claushuis TAM, Vught LA, Scicluna BP, Wiewel MA, Klouwenberg PMCK, Hoogendijk A, et al. Thrombocytopenia is associated with a dysregulated host response in critically ill sepsis patients. Blood. 2016;127(24):3062-72.

20. Levi M. Platelets in Critical Illness. Semin Thromb Hemost. 2016;42(3):252-7.

21. Stoppelaar SF, Veer C, Claushuis TAM, Albersen BJA, Roelofs JJTH, Poll T, et al. Thrombocytopenia impairs host defense in gram-negative pneumonia-derived sepsis in mice. Blood. 2014;124(25):378190.

22. Joffre J, Hellman J, Ince C, Ait-Oufella H. Endothelial responses in sepsis. Am J Respir Crit Care Med. 2020;202(3):361-370.

23. Cohen J. The immunopathogenesis of sepsis. Nature. 2002;420(6917):885-91.

24. Stéphan F, Cheffi MA, Kaplan C, Maillet J, Novara A, Fagon J, et al. Autoantibodies against platelet glycoproteins in critically ill patients with thrombocytopenia. Am J Med. 2000;108(7):554-60.

25. Guclu E, Durmaz Y, Karabay O. Effect of severe sepsis on platelet count and their indices. Afr Health Sci. 2013;13(2):333-8.

26. Liu Y, Jin G, Sun J, Wang X, Guo L. Recombinant human thrombopoietin in critically ill patients with sepsis-associated thrombocytopenia: A clinical study. Int J Infect Dis. 2020;98:144-149.

27. Jones JM, Sapiano MRP, Savinkina AA, Haass KA, Baker ML, Henry RA, et al. Slowing decline in blood collection and transfusion in the United States-2017. Transfusion. 2020;60 Suppl 2(Suppl 2):S1-S9. 
28. Aubron C, Flint AW, Bailey M, Pilcher David, Cheng AC, Hegarty C, et al. Is platelet transfusion associated with hospital-acquired infections in critically ill patients?. Crit Care. 2017;21(1):2.

29. Ninkovic S, McQuilten Z, Gotmaker R, Newcomb A, Cole-Sinclair MF. Platelet transfusion is not associated with increased mortality or morbidity in patients undergoing cardiac surgery. Transfusion. 2018;58(5):1218-1227.

\section{Tables}

Due to technical limitations, table 1 to 3 is only available as a download in the Supplemental Files section.

\section{Figures}




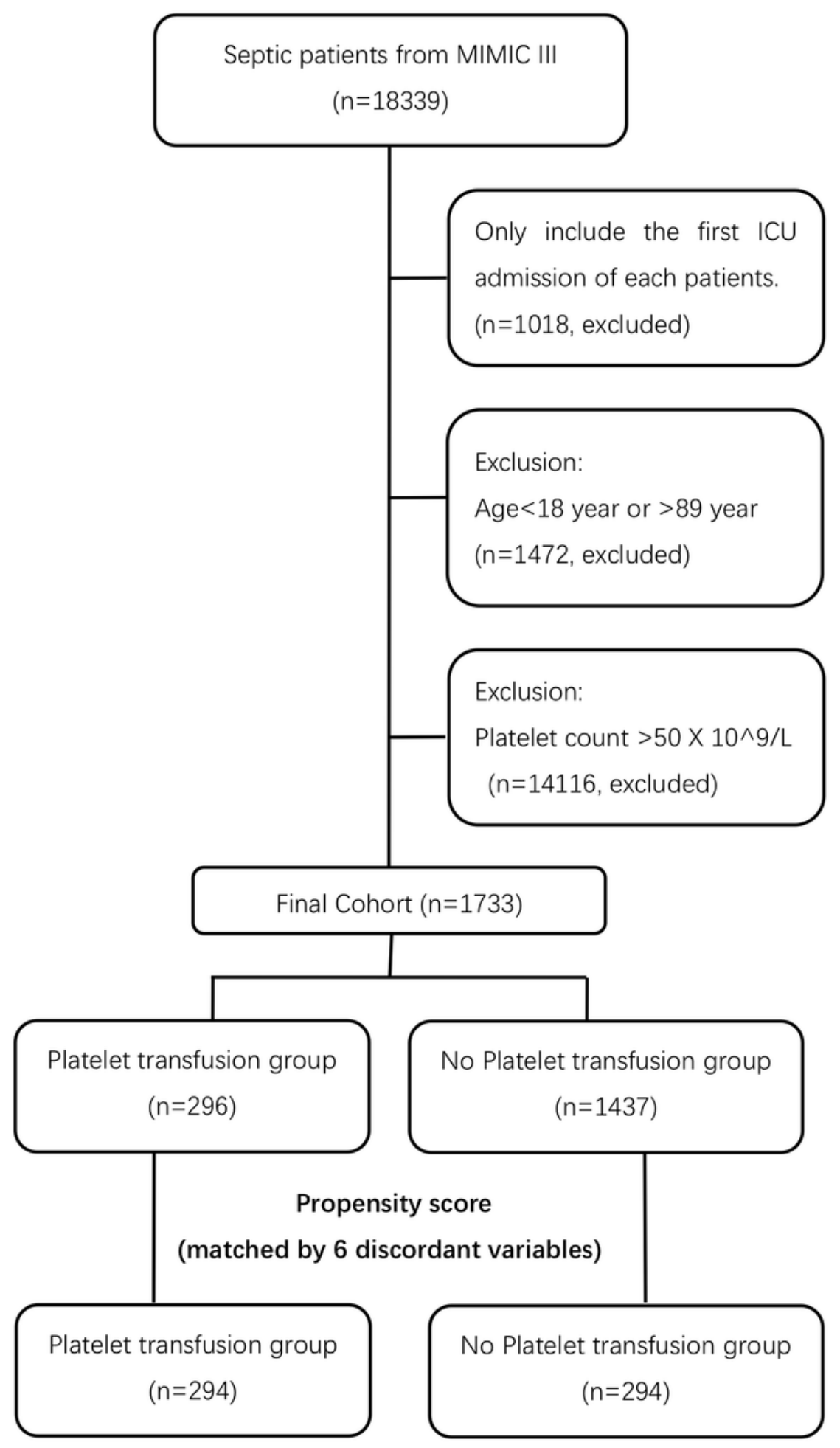

Figure 1

Flow chart of patient selection from the MIMIC III database 


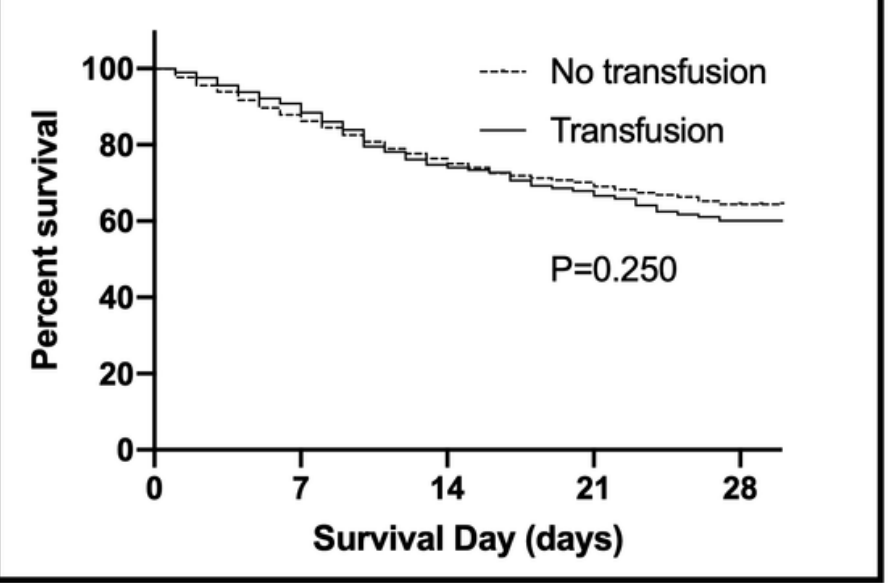

Before PSM

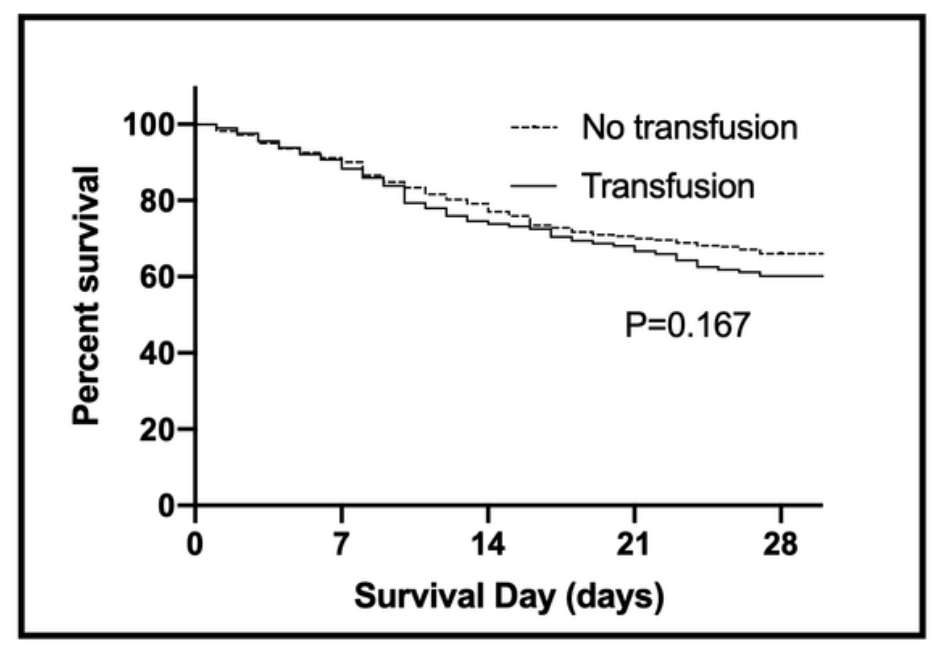

After PSM

Figure 2

The 28-day survival curves of the Platelet transfusion and No Platelet Transfusion groups before and after PSM

\section{Supplementary Files}

This is a list of supplementary files associated with this preprint. Click to download.

- Additionalfile2Table1Baseline.tiff

- Addtionalfile3Table2BeforeMatch.tiff

- Additionalfile4Table3AfterMatch.tiff 